\title{
The Neural Basis of Involuntary Episodic Memories
}

\author{
Shana A. Hall ${ }^{1}$, David C. Rubin ${ }^{1,2}$, Amanda Miles ${ }^{2}$, Simon W. Davis ${ }^{3}$, \\ Erik A. Wing ${ }^{1}$, Roberto Cabeza ${ }^{1}$, and Dorthe Berntsen ${ }^{2}$
}

\begin{abstract}
Voluntary episodic memories require an intentional memory search, whereas involuntary episodic memories come to mind spontaneously without conscious effort. Cognitive neuroscience has largely focused on voluntary memory, leaving the neural mechanisms of involuntary memory largely unknown. We hypothesized that, because the main difference between voluntary and involuntary memory is the controlled retrieval processes required by the former, there would be greater frontal activity for voluntary than involuntary memories. Conversely, we predicted that other components of the episodic retrieval network would be similarly engaged in the two types of memory. During encoding, all participants heard sounds, half paired with pictures of complex scenes and half presented alone. During retrieval, paired and unpaired sounds were presented, panned to the left or to the right. Participants in the involuntary group were instructed
\end{abstract}

\section{INTRODUCTION}

Memory theorists have long realized both the importance and difficulty of studying involuntary memories (Miller, 1962, p. 180; Neisser, 1967, p. 299). In fact, Ebbinghaus (1885) divided memory into what, in modern terms, would be called implicit memory, voluntary explicit memory, and involuntary explicit memory but did not report studies on involuntary memories - a trend that has extended to the modern era of cognitive neuroscience. In clinical syndromes, maladaptive involuntary memories have long been recognized as a key symptom of posttraumatic stress disorder (American Psychiatric Association, 2013; Horowitz, 1986). More recently, they have been described in relation to a range of other emotional disorders including depression, social phobia, bipolar disorder, and agoraphobia (Holmes \& Mathews, 2010; Williams \& Moulds, 2010; Holmes \& Bourne, 2008; Steel, Fowler, \& Holmes, 2005). Following Horowitz (1986), in the clinical literature negative involuntary (i.e., intrusive) memories are often seen to rely on a separate memory system with special properties linked to memories of the negative events (Berntsen \& Rubin, 2013). The goal of this article is to investigate the neural correlates of involuntary memory, a basic form of memory retrieval, which in its maladap-

\footnotetext{
${ }^{1}$ Duke University, ${ }^{2}$ University of Denmark, ${ }^{3}$ University of
} Cambridge to indicate the spatial location of the sound, whereas participants in the voluntary group were asked to additionally recall the pictures that had been paired with the sounds. All participants reported the incidence of their memories in a postscan session. Consistent with our predictions, voluntary memories elicited greater activity in dorsal frontal regions than involuntary memories, whereas other components of the retrieval network, including medial-temporal, ventral occipitotemporal, and ventral parietal regions were similarly engaged by both types of memories. These results clarify the distinct role of dorsal frontal and ventral occipitotemporal regions in predicting strategic retrieval and recalled information, respectively, and suggest that, although there are neural differences in retrieval, involuntary memories share neural components with established voluntary memory systems. tive forms is related to a variety of clinical syndromes. The most efficient way to accomplish this goal is to compare involuntary memories to the more thoroughly studied voluntary memories, thereby making use of and situating our findings in the general memory literature.

Involuntary episodic memories are memories of past events that come to mind spontaneously without a deliberate retrieval attempt (Berntsen, 1996). Such memories are central to our understanding of conscious mental processes; they are as frequent in daily life as intentionally retrieved memories and are functional, often directing our behavior (Berntsen, 2009, 2010). By favoring the recollection of recent events and events that share overlapping features with the ongoing situation, involuntary episodic memories operate in ways that increase the probability that they will provide information of relevance to the current situation (Berntsen, Staugaard, \& Sørensen, 2013). Involuntary memories come to consciousness with little executive control (Berntsen, 2009), and those most relevant can then be pursued in a voluntary fashion (Moscovitch, 1995). They are the remindings that guide daily life (Hintzman, 2011; Berntsen, 2009; Schank, 1999) and may be the evolutionary precursor of voluntary episodic memory (Martin-Ordas, Berntsen, \& Call, 2013; Berntsen, 2009; Donald, 1991).

Although laboratory investigations of the neural basis of involuntary memories have been limited, recent advances 
in neuroimaging allow for the possibility of assessing the neural processes supporting involuntary memories, independent of any overt response demands. Here, we present a neuroimaging study specifically designed to aid in understanding the neural basis of involuntary episodic memories. In our view, three types of measurements are needed. The first is simultaneous event-related functional imaging data to examine the neural basis of this behavior, which may indicate that mnemonic details were retrieved, despite the lack of an overt response. The second is a postscan behavioral measure to confirm that an accurate involuntary memory was recalled. The third is a postscan report that voluntary effort did not occur or was minimal. By its nature, this report must be a private phenomenological report, but one that should be confirmed by observable phenomena. Studies in which participants report involuntary memories as they occur have been successful in producing replicable and theoretically informative results. However, reporting on involuntary memories as they occur necessarily involves a voluntary search after the involuntary memory comes to mind to judge whether the involuntary memory is accurate and should be reported. This makes it difficult to dissociate neural activity due to involuntary memory retrieval from neural activity due to voluntary postretrieval processes.

In addition, if involuntary memories are to be compared with voluntary memories, the study should be a betweensubjects design. If participants were instructed to do both voluntary and involuntary retrieval, it is unclear whether participants would be able to switch strategies across trials and refrain from using voluntary retrieval strategies during involuntary memory trials. Again, this has the potential to confound neural activity due to involuntary retrieval with neural activity due to voluntary retrieval strategies. Thus, we intend not only to report theoretically interesting results but also to propose a basic design that can be modified to study the neural basis of many aspects of involuntary memories in healthy and clinical populations while avoiding the most obvious experimental confounds.

Researchers have found many similarities between voluntary and involuntary memories. Specifically, they both tend to be emotionally positive rather than negative, are more frequent for emotionally intense events, show a similar forgetting curve, happen equally as often in daily life, and show similar distributions to one another across the life span (Berntsen \& Rubin, 2013; Rasmussen \& Berntsen, 2011; Berntsen, 2009; Rubin \& Berntsen, 2009). However, there is one important difference: Voluntary memories require effortful strategic retrieval operations, whereas involuntary memories do not. Thus, the most parsimonious way to approach the investigation of the neural basis of involuntary memory is to assume involuntary memories rely on the same neural correlates as voluntary memories, except for processes involved in voluntary strategic retrieval. That is, we assume that involuntary and voluntary memories rely on the same encoded information and differ only in how that information is retrieved (Berntsen,
2009). This hypothesis is also the most informative, as we already know a great deal about the neural basis of voluntary memory and this knowledge can provide considerable specificity to our predictions if identical encoding conditions are used in an experiment. Existing studies of the neural basis of involuntary memories also have used this strategy. Thus, the logic is to assume similarity except for differences in retrieval and note any exceptions that may arise as hypotheses for future study.

We predict that the primary neural difference between voluntary and involuntary memories will be in the pFC. Although episodic memory researchers originally interpreted $\mathrm{pFC}$ contributions in terms of processes specific to episodic memory, it is now generally accepted that the processes contributed by pFC to episodic retrieval are control processes shared with other cognitive tasks, such as working memory (Cabeza, Dolcos, Graham, \& Nyberg, 2002) and decision-making (Fleck, Daselaar, Dobbins, \& Cabeza, 2006).

Whereas the left pFC has long been associated with both verbal working memory operations (D'Esposito, Postle, \& Rypma, 2000) and controlled semantic retrieval (Thompson-Schill, D’Esposito, Aguirre, \& Farah, 1997), there are regions in the lateral $\mathrm{pFC}$ that are associated with controlling strategic retrieval operations in episodic retrieval (Mitchell \& Johnson, 2009; Buckner \& Wheeler, 2001). For example, compared with item recognition tasks, source memory tasks elicit greater activity in left dorsolateral pFC (DLPFC; Dobbins \& Han, 2006; Dobbins \& Wagner, 2005). Importantly, in contrast to neighboring ventrolateral pFC (O'Connor, Han, \& Dobbins, 2010; Vilberg \& Rugg, 2008), studies that report left DLPFC activity for contextual retrieval find that activations in this region do not differ for successful and unsuccessful retrieval trials (Meltzer \& Constable, 2005; Dobbins, Rice, Wagner, \& Schacter, 2003; Dobbins, 2001), suggesting that DLPFC is associated with strategic retrieval, not with retrieval success. Conversely, a constellation of more posterior and ventral cortical regions, including the medial temporal lobe (MTL), the posterior midline cortex (retrosplenial cortex, posterior cingulate), ventral parietal cortex (VPC), and sensory reactivation areas, is reliably more active for successful than unsuccessful retrieval independent of how a particular memory trace is activated (Habib \& Nyberg, 2008). These regions likely form the neural correlates for involuntary as well as voluntary memory retrieval, with voluntary memories activating additional regions associated with strategic retrieval.

Only two functional imaging studies have examined involuntary memories in this context. The first study paired a picture with a word describing the main contents of the picture during encoding (Hall, Gjedde, \& Kupers, 2008). During recall, participants saw the words and made a judgment about whether the item could be worn. The blocks alternated between involuntary retrieval, in which participants were not told to try to recall the picture that had been paired with the word, and 
voluntary retrieval, in which they were told to recall the picture. Among other findings, the results showed that both voluntary and involuntary memories activated $\mathrm{pFC}$ regions. One limitation of this study is the use of a within-subjects design with alternative involuntary and voluntary blocks. pFC activations during involuntary memory could reflect a contamination by voluntary retrieval strategies as discussed above.

In the other study of involuntary memory, a combined event-related fMRI and EEG study by Kompus, Eichele, Hugdahl, and Nyberg (2011), participants were presented with sounds and pictures of objects during encoding. In the voluntary memory condition, participants were shown a word and were asked to try to recall whether the word described an object presented during encoding and whether the word was concrete or abstract. During the involuntary memory condition, participants were asked to decide whether the word was concrete or abstract. They also were asked, however, to press a button if they had a spontaneous memory of the object when they saw the word. Imaging results in this study suggested that voluntary memories rely on DLPFC to a greater extent than involuntary memories. In contrast, successful memories in both conditions showed a general overlap in neural recruitment. Although this study provides strong eventrelated evidence that involuntary and voluntary memories share a distinct, but overlapping, neural signature, it also has the limitation of a within-subject design. Moreover, because participants had to press a key when they had an involuntary memory, having episodic memories became in practice a secondary goal of the task. Because there was a dedicated response for having associated memories, it is likely that participants checked the accuracy of these memories before responding, thereby introducing a voluntary memory component in the task. Thus, the differences in activity found could be explained in quantitative terms by greater strategic retrieval in the voluntary condition rather than in qualitative terms.

In the current study, we sought to address the issues in the previous studies by having a between-subjects design in which participants in the involuntary group were not told that they were in a memory experiment, and there was no online reporting of involuntary memories so there was no expectation that they may have felt compelled to fulfill. We adapted to fMRI a behavioral paradigm that successfully elicits involuntary memories (Berntsen et al., 2013). During an encoding session (see Figure 1), participants heard a series of environmental sounds (e.g., doorbell ringing). Half of the sounds were presented alone (unpaired sounds), whereas the other half were paired with pictures of complex scenes (paired sounds), which participants tried to associate with the sounds. During both voluntary and involuntary retrieval in the fMRI scanner, paired and unpaired sounds were presented, panned to either the left or right. Participants in the involuntary condition were asked to decide from which side the sound originated, whereas participants in the voluntary condition did the same task and were also asked to recall the corresponding picture from encoding if there had been one. After the scan, participants heard all of the sounds and identified those for which they experienced a memory in the scanner and tried to describe the associated picture in detail, allowing us to analyze only the trials in which a memory was recalled.

We made two predictions. First, we predicted that brain regions associated with successful retrieval would be similar for voluntary and involuntary memories. Specifically, in both the voluntary and involuntary conditions, we expected that paired sounds compared with unpaired sounds would elicit greater activity in a network of regions associated with successful memory retrieval, including the MTL, retrosplenial cortex, and posterior cingulate (Mitchell \& Johnson, 2009), VPC (Cabeza, Ciaramelli, Olson, \& Moscovitch, 2008; Ciaramelli, Grady, \& Moscovitch, 2008; Vilberg \& Rugg, 2008; Wagner, Shannon, Kahn, \& Buckner, 2005), and sensory reactivation areas (Danker \& Anderson, 2010). Given that the sensory information retrieved for paired compared with unpaired sounds was visual (memory for the pictures), we predicted reactivation activity in visual cortex. Second, we predicted that brain regions generally associated with cognitive control (Miller, 2000) and more specifically with strategic retrieval, such as lateral $\mathrm{pFC}$ (Mitchell \& Johnson, 2009; Dobbins et al., 2003; Dobbins, Foley, Schacter, \& Wagner, 2002), would show greater activity in the voluntary memory group than the involuntary memory group. In summary, we expected a dissociation between regions often associated with retrieval success, namely MTL, retrosplenial cortex, posterior cingulate regions, VPC, and sensory reactivation areas, which would be shared for voluntary and involuntary memory, and lateral pFC, which would be active for both paired and unpaired sounds in the voluntary memory group but not the involuntary memory group.

\section{METHODS}

\section{Participants}

Twenty-nine participants (18 women, mean age $=26$ years, range $=18-40$ years) were recruited through e-mail and through the Brain Imaging and Analysis Center at Duke University. All participants were right-handed, native English speakers with normal or corrected-to-normal vision. Three participants were excluded because of excessive movement during the fMRI session (any scans with $>5 \mathrm{~mm}$ or more than $6 \%$ of their total scans with between 3 and $5 \mathrm{~mm}$ ). Participants had no past or current neurological or psychiatric disorders. The between-subjects design had 14 participants in the involuntary group (eight women, six men, mean age $=25$ years, range $=19-35$ years) and 12 in the voluntary group (eight women, four men, mean age $=28$ years, range $=21-35$ years). Participants gave written informed consent for a protocol approved by the Duke University Institutional Review Board. 


\section{Session 1: Before Scanning}

\section{A}

\section{Paired sound encoding (outside the scanner)}

Participants associated sounds and scenesEncoding repeated 3 times
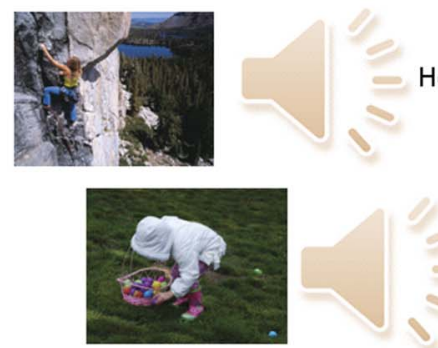

Eggs cracking
Session 2: During and After Scanning

C

Paired sound encoding (in-scanner)

Participants rated how strongly sound and picture were linked

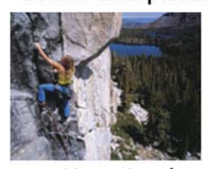

Unpaired sound encoding (in-scanner)

Participants rated sound uniqueness

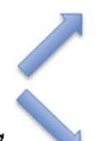

$D$ Involuntary in-scanner retrieval

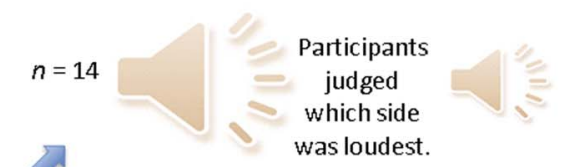
to recall the picture.

$\longrightarrow$

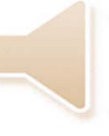

B Unpaired sound encoding

(outside the scanner)

Participants rated

sound uniqueness

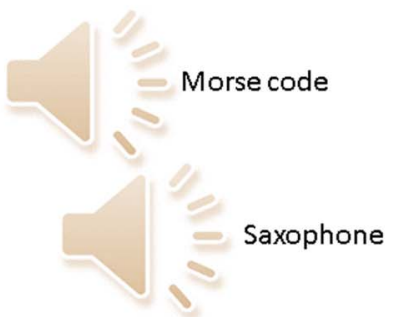

\section{E Postscan questions}

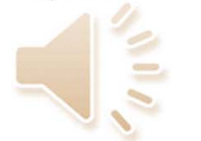

Voluntary in-scanner retrieval

Participants judged which side was loudest and tried

Saxophone
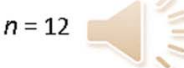
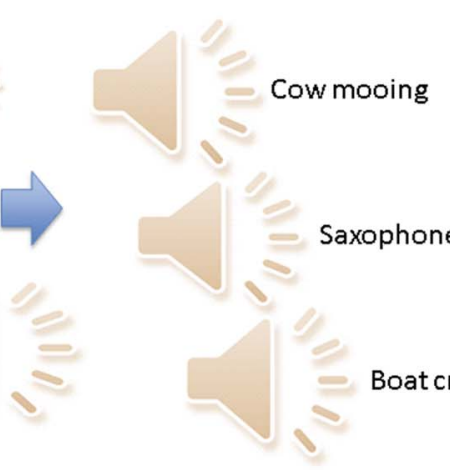

Boat creaking

Figure 1. Experimental design. (A) During prescan encoding, participants heard a sound paired with a picture and were asked to type a sentence linking the sound and picture. (B) During another prescan encoding session, participants heard unpaired sounds and rated the uniqueness of the sound. (C) On Day 2, participants performed a "re-encoding" session in the scanner, whereupon they were presented with previous sound-picture pairs and asked to rate on an 8-point scale how well the sound and picture went together based on the story they had formulated previously; with the unpaired sounds, they were asked how distinguishable the sounds were from the other sounds. (D) Participants were subsequently divided into either involuntary or voluntary memory groups. The involuntary group heard both paired and unpaired sounds and was asked to judge on which side the sound was located, whereas the voluntary group was additionally told to also try to remember the picture that had been paired with the sound during encoding, if there had been one. (E) After the scan, all participants heard all sounds and were asked questions about their experiences in the scanner (see text for postscan questionnaire details).

\section{Materials}

We used 50 scenes and 100 environmental sounds (e.g., dog panting, doorbell ringing). The images and sounds were obtained from multiple sources including the International Affective Picture System (Lang, Bradley, \& Cuthbert, 2005) for the pictures and the SUN database (groups.csail.mit.edu/vision/SUN/) for the sounds. All sounds were calibrated to be of equal volume and equal duration $(4 \mathrm{sec})$. As part of the retrieval manipulation, versions of the sounds were produced, in which the sounds were panned $15 \%$ to the left or to the right.

\section{Experimental Design and Procedure}

\section{Session 1: Two Days before Scanning}

Two days before scanning, participants encoded paired and unpaired sounds outside the scanner (see Figure 1).
To disguise the later involuntary memory task, participants were given the cover story that the study was investigating the effects of pairing sounds with pictures on a later sound laterality task (in the scanner). All encoding trials were self-paced. First, participants encoded 50 paired sounds. Each sound was presented with a picture $(4 \mathrm{sec})$ and, to ensure strong memory associations, the entire 50 pair list was encoded three times: (i) each sound was presented simultaneously with a picture and participants typed a sentence integrating the pair into a plausible story, (ii) each sound-picture pair was presented with the subject-generated sentence and participants edited the sentence if they could improve it, and (iii) each sound was presented alone immediately preceding the soundpicture pair, and participants were to recall the associated sentence and imagine the corresponding picture. An attempt was made to show pictures with some semantic relevance to the sound, but that could not be described 
by simply describing the sound. This was done so that when participants were later asked to describe the pictures from memory, it would not be possible for a description of the sound to be confused with a description of the picture. We piloted to make sure the sound-picture associations could be made and that recall would include information that was clearly not in the sound alone. After encoding the sound-picture pairs, participants encoded 50 unpaired sounds. We blocked the paired and unpaired sounds to separate them and thereby improve source monitoring of which sounds had and did not have associated pictures. We used the paired-unpaired sound order because it allowed us to have participants rate each unpaired sound on an 8-point scale how distinguishable the sound was compared with other sounds encountered in the study, thereby further decreasing the likelihood of the participant confusing paired and unpaired sounds and later erroneously recalling pictures to the unpaired sounds. This rating scale required the unpaired sounds to be presented after all paired sounds. We accepted this lack of randomization because it was unlikely to interact with our hypotheses.

\section{Session 2: During and After Scanning}

The fMRI session consisted of the re-encoding of paired and unpaired sounds (two runs) followed by the critical memory recall task (two runs). Before the re-encoding runs, participants briefly practiced the recall task described below to ensure that participants were comfortable with the responses and the pace. During the re-encoding runs, each stimulus was presented for $4 \mathrm{sec}$ and followed by a 4 -sec response window and then by a fixation period (jittered with a mean of $4 \mathrm{sec}$ ). The fixation period served as the implicit baseline against which task-related BOLD activity was measured. In the re-encoding of paired sounds, the 50 sound-picture pairs were presented, and participants rated on an 8-point scale how well the sound and picture went together based on the story they had formulated previously. In the re-encoding of unpaired sounds, the 50 sounds were presented, and participants made the same distinguishability judgment they had made previously.

During the recall runs, the 50 paired and 50 unpaired sounds (randomly intermixed) were presented, panned $15 \%$ to either the left or the right using specialized audio software (Audacity, audacity.sourceforge.net/). Participants were instructed to use their index finger to press the button corresponding to the side on which the sound was louder (i.e., the direction from which the sound seemed to originate). Each sound was presented for $4 \mathrm{sec}$, followed by a 2-sec response window and a jittered fixation period with a mean of $4 \mathrm{sec}$. Participants were instructed at the beginning of the run that they must wait until after the sound had ended to make their response. The sound lateralization task was thus matched across voluntary and involuntary groups. In addition to performing the spatial discrimination task, participants in the voluntary group were asked to recall the pictures that had been paired with the sound with as many of the details as possible. Participants in the involuntary group were told that having an image come to mind spontaneously was fine but they should not try to recall the images intentionally. This instruction was necessary because pilot evidence suggested that some participants in the involuntary group might try to recall pictures even though the instructions did not require it. After the instruction was added, effort ratings decreased in the involuntary group.

\section{Post Scanning Phase}

Immediately following the scanning session, participants completed a postscanning questionnaire to assess their memory for the pictures. At a computer terminal outside the scanning room, participants were presented with all 100 sounds (50 paired and 50 unpaired, randomly intermixed) on stereophonic headphones. After the presentation of each sound, participants were asked whether they recalled an image during the scan (yes, no), how vivid the image was during the scan $(1=$ not at all vivid, $8=$ very vivid), and how hard they tried to recall an image during the scan $(0=$ did not try at all, $7=$ tried very hard $)$.

Next, the 50 paired sounds were presented, and participants were asked to provide a description of the picture originally presented with the sound. This was to provide a final check that the image that participants recalled as being originally paired with a sound was indeed of the original picture. Three independent raters scored the similarity between the original picture and the description based on information than what could not be guessed on the basis of the sound alone $(1=$ no similarity to picture, $10=$ exact/bighly detailed description). The scores of all three raters were then averaged for each sound for each participant. The only scores that were analyzed were scores for pictures in which participants reported having a memory in the scanner.

\section{Image Acquisition and Preprocessing}

Imaging was conducted on a 3T GE Signa Excite MRI scanner (GE Healthcare, Waukesha, WI) with an eightchannel head coil. Head motion was minimized with foam pads and a headband, and participants wore earplugs to reduce scanner noise. The imaging sequence included a 3-D plane localizer, followed by T1-weighted structural image and four runs of $\mathrm{T} 2 *$-weighted (functional). The two encoding runs were followed by two retrieval runs; a resting state scan $(360 \mathrm{sec})$ was acquired before the first encoding run and after the final retrieval run. Slice orientation was near-axial, parallel to the anterior-posterior commissure plane. The T1-weighted anatomical images were 60 contiguous slices acquired with a high-resolution, 3-D fast inverse recovery-prepared spoiled gradient recalled sequence, with repetition time $(\mathrm{TR})=7.3 \mathrm{msec}$, echo 
time $=2.97 \mathrm{msec}$, inversion recovery time $=450 \mathrm{msec}$, field of view $=256 \mathrm{~mm}, 2 \mathrm{~mm}$ slice thickness, flip angle $=$ $12^{\circ}$, voxel size $=2 \times 2 \times 2 \mathrm{~mm}, 256 \times 256$ matrix, and a parallel imaging with a selection factor of 2 . The $\mathrm{T} 2 *_{\text {- }}$ weighted echo-planar, functional images were sensitive to the BOLD signal. These were 30 contiguous slices acquired using an inverse spiral sequence, with TR $=2000 \mathrm{msec}$, echo time $=30 \mathrm{msec}$, field of view $=256 \mathrm{~mm}, 4 \mathrm{~mm}$ slice thickness, flip angle $=60^{\circ}$, voxel size $=4 \times 4 \times 4 \mathrm{~mm}$, and $64 \times 64$ matrix.

Preprocessing and analyses of functional imaging data were conducted with Statistical Parametric Mapping software (SPM5; Wellcome Department of Cognitive Neurology, London, UK), along with locally developed Matlab (Mathworks, Natick, MA) scripts. The first three volumes of each run were discarded. Images were corrected for slice-timing and head motion, spatially normalized to the Montreal Neurological Institute template, and then spatially smoothed with an 8-mm Gaussian kernel. A high-pass filter was included in every model to correct for scanner drift. Participants with between 3 and $5 \mathrm{~mm}$ of movement in $6 \%$ or fewer of their scans were corrected with Artrepair (cibsr.stanford.edu/tools/ArtRepair/ArtRepair.htm).

\section{fMRI Data Analysis}

fMRI results were analyzed using SPM5. The first level model included regressors for paired sound trials (excluding those for which no picture memory was reported after scanning) and unpaired sound trials (excluding those for which a picture memory was reported after scanning). These trials were split according to sound lateralization (paired left, paired right, unpaired left, and unpaired right) to account for the additional variance due to the difference in sound laterality, but this factor was collapsed at the second level. As nuisance regressors, the model also included paired sound trials with no picture memories and unpaired sound trials with picture memories, as well as motion and run regressors.

Activations were identified using a finite impulse response (FIR) model, which was preferred over a standard canonical hemodynamic response (HDR) model to better understand group effects. An examination of the main effects of group or condition should reveal activity that is sustained over the entire trial. An examination of the interaction between group or condition and time should reveal activity that changes with time. Voluntary and involuntary memory groups performed different tasks; hence, they could differ not only in transient (event-related) but also in more sustained (task-related) activations. Because we could not formulate clear and theoretically defensible hypotheses of the time courses of activation, we used FIR analyses throughout our main analyses. Other studies have used FIR to differentiate between sustained and transient activity (Preuschhof, Heekeren, Taskin, Schubert, \& Villringer, 2006; Corbetta, Kincade, Ollinger, McAvoy, \& Shulman, 2000) and have found sustained activity in a variety of regions in response to sustained stimuli or cognitive responses (Courtney, Ungerleider, \& Keil, 1997). In contrast to the group effects, condition effects (paired vs. unpaired) can only occur after the sound cue is presented; hence, they should show a canonical HDR. Because we investigate both types of effects within the same model, for condition effects we focus exclusively on effects that showed a significant interaction with time (TRs), whereas for group effects we also considered main effects of group. In all cases, the significance threshold was $p<.001$ (uncorrected) with a minimum cluster size of 10 contiguous voxels.

\section{RESULTS \\ Behavioral Results}

During the scan, both groups performed equally well on the laterality task (voluntary correct: $67.50 \%, S D=$ 11.00\%; involuntary correct: $69.86 \%, S D=8.04 \%, t(24)=$ $-0.63, p>.50$ ), indicating that the level of attentiveness was similar between the groups during the recall block. Postscan questionnaires were administered to fully characterize and verify that participants did in fact undergo the expected mnemonic experience. Critically, participants were first asked whether they recalled mnemonic details (voluntarily or involuntarily) for each sound played during the recall phase. There was a main effect of Group, $F(1,24)=7.75, p<.05$, and Condition, $F(1,24)=268.68$, $p<.001$, on self-reported recall, and an interaction, $F(1$, $24)=7.59, p<.05$, such that voluntary participants recalled a greater proportion of memories of the pictures from paired sound trials $(94.50 \%, S D=5.92 \%)$ than did involuntary participants $(68.71 \%, S D=31.11 \%, t(14.10)=$ $3.04, p<.001)$, but there was no difference in the pictures that were incorrectly recalled to unpaired sound trials $(3.83 \%, S D=4.22 \%$ vs. $4.14 \%, S D=4.74 \%$, respectively). Participants were also asked to report how effortful they found retrieving each associated picture for each sound. There was a main effect of Group, $F(1,24)=$ $60.12, p<.001$, and a main effect of Condition, $F(1,24)=$ $5.59, p<.05$, and no significant Group $\times$ Condition interaction on these effort ratings, $F(1,24)=3.40, p>.05$. On a $0-7$ retrieval effort scale, voluntary memory participants reported an average retrieval effort of $4.20(S D=2.13)$ for the paired sounds and $2.33(S D=2.02)$ for the unpaired sounds. Involuntary memory participants reported an average retrieval effort of $0.49(S D=0.74)$ for the paired sounds and $0.26(S D=0.45)$ for the unpaired sounds. The participants had considerable training on the paired and unpaired sounds, so the increased effort for paired sounds compared with unpaired sounds in the voluntary condition most likely reflects their knowledge of whether retrieving a picture was possible. The large difference in retrieval effort between voluntary and involuntary groups combined with the differences in their reports of the number of sounds that led to recall of 
mnemonic details lends support to the effectiveness of the retrieval manipulation.

Ratings of vividness were made for each recalled picture on a scale from 1 to 8 . There was no Group $\times$ Condition interaction for vividness, $F(1,24)=2.50, p>.10$, but there was a main effect of Group, $F(1,24)=12.06$, $p<.005$, and a main effect of Condition, $F(1,24)=$ $115.70, p<.001$. Consistent with their instructions to recall the image with as many details as possible, the voluntary group reported higher vividness ratings $(M=$ $4.19, S D=0.41)$ than the involuntary group $(M=3.00$, $S D=1.15)$. The recalled pictures from the paired sounds elicited higher vividness ratings $(M=5.66, S D=1.96)$ than the pictures that were incorrectly recalled to the unpaired sounds $(M=1.42, S D=0.79)$ for all trials in which an image was recalled.

Finally, participants wrote descriptions of the pictures, which were rated by three independent raters for similarity to the picture presented during encoding. Descriptions typically ranged from one to two sentences with two to three descriptive elements. There was no significant difference between similarity ratings of the descriptions of the pictures between the groups, $t(24)=1.12, p=.27$. Interrater reliability was high $(\alpha=0.95)$. The average rating for the voluntary participants was $9.31( \pm 0.72)$, and the average rating for the involuntary participants was $9.54( \pm 0.27)$. This suggests that the sound-picture pairings were learned well and that the difference in the number of sounds that evoked a memory was not because of a difference in the participants' ability to recall the pictures.

\section{fMRI Results}

Table 1 lists regions showing differences of Condition (paired vs. unpaired sounds) $\times$ Time, main effects of Group, and Group (voluntary vs. involuntary memory) $\times$ Time. All interactions with time were conducted with all

Table 1. Brain Regions Showing Differences as a Function of Condition or Group $(p<.001$, Cluster Size $=10)$

\begin{tabular}{|c|c|c|c|c|c|c|c|}
\hline Region & $B A$ & Lat & Voxel Count & $x$ & $y$ & $z$ & $F$ \\
\hline \multicolumn{8}{|c|}{ Condition (Paired $>$ Unpaired) $\times$ Time } \\
\hline Hippocampus & & $\mathrm{L}$ & 776 & -26 & -34 & -4 & 6.39 \\
\hline \multirow[t]{2}{*}{ Parahippocampal cortex } & 36 & $\mathrm{~L}$ & & -26 & -30 & -19 & 6.98 \\
\hline & 30 & $\mathrm{R}$ & & 11 & -41 & 4 & 10.52 \\
\hline Retrosplenial cortex & 30 & $\mathrm{R}$ & & 8 & -53 & 15 & 8.95 \\
\hline Posterior cingulate gyrus & 31 & $\mathrm{~L}$ & & -8 & -34 & 30 & 7.63 \\
\hline \multirow[t]{2}{*}{ Precuneus } & 31 & $\mathrm{~L}$ & & -4 & -60 & 34 & 7.50 \\
\hline & 31 & $\mathrm{R}$ & & 4 & -64 & 34 & 7.80 \\
\hline \multirow[t]{2}{*}{ Angular gyrus } & 39 & $\mathrm{~L}$ & & -49 & -71 & 30 & 7.27 \\
\hline & 39 & $\mathrm{~L}$ & & -49 & -68 & 23 & 7.38 \\
\hline Superior parietal lobule & 7 & $\mathrm{~L}$ & & -30 & -71 & 49 & 6.24 \\
\hline Thalamus & & $\mathrm{L}$ & & -15 & -34 & 0 & 7.37 \\
\hline Visual cortex & 19 & $\mathrm{~L}$ & & -38 & -79 & 34 & 8.00 \\
\hline \multirow[t]{2}{*}{ Superior temporal gyrus } & 22 & $\mathrm{~L}$ & 44 & -53 & -15 & 4 & 6.85 \\
\hline & 22 & $\mathrm{R}$ & 144 & 60 & -8 & 4 & 7.22 \\
\hline Auditory cortex & $41 / 42$ & $\mathrm{R}$ & & 49 & -23 & 11 & 5.36 \\
\hline Middle temporal gyrus & 20 & $\mathrm{R}$ & & 53 & -4 & -23 & 4.42 \\
\hline Cerebellum & & $\mathrm{R}$ & 17 & 11 & -68 & -19 & 4.93 \\
\hline \multicolumn{8}{|c|}{ Group (Voluntary > Involuntary) } \\
\hline DLPFC (middle frontal gyrus) & 9 & $\mathrm{~L}$ & 11 & -38 & 11 & 38 & 3.68 \\
\hline \multicolumn{8}{|c|}{ Group (Voluntary $>$ Involuntary) $\times$ Time } \\
\hline Auditory cortex & $41 / 42$ & $\mathrm{~L}$ & 13 & -45 & -26 & 19 & 6.21 \\
\hline
\end{tabular}

$\mathrm{BA}=$ Brodmann's area Lat $=$ lateralization $; x y z=$ coordinates. 
seven time points. Consistent with our first prediction, there were significant differences across the voluntary and involuntary groups between paired and unpaired sounds. In Figure 2A, activity from the Condition $\times$ Time interaction is shown, in part to make clearer the extent of the large 776-voxel cluster shown in Table 1. This large ROI was masked with MTL, posterior midline, ventral parietal, and visual regions, respectively, using ROIs from the Wake Forest University PickAtlas toolbox (www.fmri. wfubmc.edu/download.htm), and significant voxels within those masks are shown in Figure 2B. The time courses in Figure $2 \mathrm{C}$ represent the difference in activity for pairedunpaired sounds for four sub-ROIs over seven time points. The time courses in Figure 2D represent activity for all four conditions (voluntary paired sounds, voluntary unpaired sounds, involuntary paired sounds, and involuntary unpaired sounds) separately. Parahippocampal gyrus, posterior cingulate, and precuneus were also active in a conjunction analysis between voluntary paired $>$ unpaired sounds and involuntary paired $>$ unpaired sounds (see Table 2). The joint probability of the conjunction map was $p<.001$. None of these regions showed significant interactions with group, indicating that they were common for voluntary and involuntary memory. As illustrated by Figure 2C, paired $>$ unpaired differences largely overlapped and had a very similar time course for the voluntary and involuntary conditions. No region showed greater activity for unpaired than paired sounds.

Consistent with our second prediction, left DLPFC (middle frontal gyrus) showed greater activity for volun-

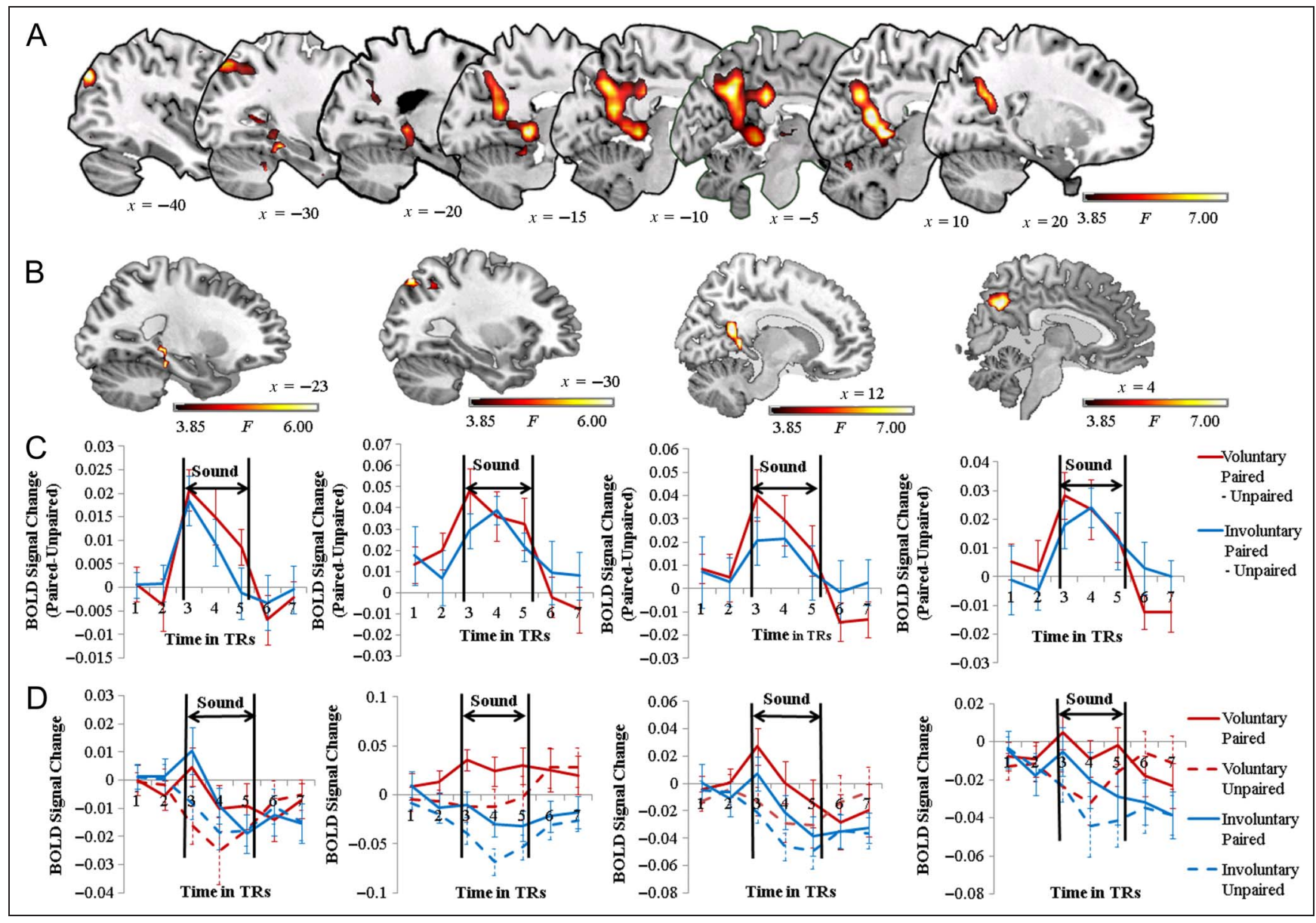

Figure 2. Effects of condition on voluntary and involuntary memory recall. Brain images display significant activations at $p<.001,10$ voxels. (A) Sagittal views of brain activity from the Condition $\times$ Time interaction. This broad posterior activation pattern demonstrates a pattern of activation typical of voluntary memory retrieval, suggesting that voluntary and involuntary memory retrieval recruit the same regions. (B) From left to right, selective clusters in the hippocampus and parahippocampal cortex, left angular gyrus, posterior midline (including posterior cingulate gyrus and retrosplenial cortex), and visual cortex. Clusters were produced by masking the large 776 voxel cluster above with the ROIs of MTL, inferior parietal cortex, posterior midline regions, and visual regions, respectively, derived from the Wake Forest University PickAtlas. (C) Graphs plot the estimated FIR response difference between paired and unpaired sounds for the voluntary (red) and the involuntary (blue) groups within each cluster of activity shown above (B). The activity between the bold vertical lines indicates activity correlating with the peak of activity expected for the sound assuming a normal HDR. Significant differences between paired and unpaired trial activity in these regions demonstrate that these are regions that are active more during trials in which there is a memory. Similarity between the voluntary and involuntary groups demonstrates that this activity is similar between groups. (D) Same data as above, divided to show the estimated FIR response for the voluntary paired sounds, voluntary unpaired sounds, involuntary paired sounds, and involuntary unpaired sounds separately. 
Table 2. Brain Regions Showing Similarities in a Voluntary Paired $>$ Unpaired $\times$ Time and Involuntary Paired $>$ Unpaired $\times$ Time Conjunction Analysis $(p<.001$, Cluster Size $=10)$

\begin{tabular}{|c|c|c|c|c|c|c|c|}
\hline Region & $B A$ & Lat & Voxel Count & $x$ & $y$ & $z$ & $F$ \\
\hline Posterior cingulate & 30 & $\mathrm{R}$ & 25 & 8 & -53 & 15 & 6.94 \\
\hline Posterior cingulate & 30 & $\mathrm{~L}$ & & -11 & -56 & 15 & 5.14 \\
\hline Precuneus & 7 & $\mathrm{R}$ & 43 & 4 & -64 & 34 & 6.76 \\
\hline Precuneus & 7 & $\mathrm{~L}$ & & -11 & -64 & 34 & 3.85 \\
\hline Parahippocampal gyrus & 27 & $\mathrm{~L}$ & 20 & -15 & -38 & 0 & 5.94 \\
\hline Superior temporal gyrus & 22 & $\mathrm{R}$ & 10 & 56 & -11 & 0 & 5.4 \\
\hline Superior temporal gyrus & 22 & $\mathrm{~L}$ & 10 & -53 & -15 & 4 & 4.32 \\
\hline Middle temporal gyrus & 21 & $\mathrm{~L}$ & & -56 & -11 & -4 & 4.14 \\
\hline Superior parietal lobule & 7 & $\mathrm{~L}$ & 10 & -30 & -71 & 49 & 5.33 \\
\hline Posterior cingulate & 23 & $\mathrm{~L}$ & 16 & -8 & -34 & 34 & 4.28 \\
\hline Posterior cingulate & 31 & $\mathrm{~L}$ & & -8 & -45 & 30 & 3.85 \\
\hline
\end{tabular}

$\mathrm{BA}=$ Brodmann's area Lat $=$ lateralization; $x y z=$ coordinates.

tary than involuntary memory groups with no difference between paired and unpaired sounds. As illustrated by Figure 3B, the voluntary-involuntary difference can be observed at all TRs including 1 and 7 , indicating a sustained effect that persisted during the intertrial interval. This pattern suggests that participants in the voluntary group maintained a controlled strategic retrieval throughout the task. The only other region that showed a significant group effect was the left auditory cortex, which suggests greater top-down attention to the sound in the voluntary than the involuntary group. This effect showed an interaction with time at $p<.001$, because it was timelocked to the sound presentation and disappeared by the end of the trial. No region showed greater activity for the involuntary than the voluntary memory group, nor were there other regions showing differences in either the main effect of Group or the Group $\times$ Time interaction, as can be seen in Table 1 .

The design in this study could not ensure that the between-group difference in DLPFC during retrieval was not because of spontaneously occurring group differences. To address this, we compared activity during encoding. During encoding, participants did not know to which group they had been assigned, and there had not yet been any differences in the instructions. Analyses within the DLPFC cluster from the between-group analysis revealed no difference between groups at $p<.05$ with a cluster size of 1 . Looking bilaterally within middle frontal gyrus, superior frontal gyrus, superior OFC, and middle OFC as defined by the PickAtlas toolbox (www.fmri.wfubmc.edu/ download.htm) at $p<.01$ with a cluster size of 10 , there was also no difference between groups. Finally, at $p<$ .01 with a cluster size of 1 in these same frontal regions, there were only 17 total voxels active out of a total of 2178 voxels, whereas 22 would be expected by chance.
Thus, the differences in the DLPFC during retrieval could not have been a carryover of encoding differences.

\section{Post hoc Analyses: Mnemonic Success}

Our initial analysis of the Paired Status $\times$ Time interaction provided a clear set of posterior temporal and midline parietal cortices demonstrating activation greater in the paired than unpaired conditions. However, because memory retrieval analyses were conducted only on recalled pictures for paired stimuli, we performed a post hoc FIR analysis of trials for which participants reported experiencing a memory during retrieval versus trials in which they did not. This analysis was done at the group level. The only trials analyzed were those in which the postretrieval description of the picture provided by the participants was judged to be accurate. We created a mask from the Paired Status $\times$ Time analysis (to investigate whether there was an overlap with regions associated with retrieval success) and from the main effect of Group analysis (to investigate whether there was an overlap with regions associated with strategic retrieval) within which we examined activity at the group level. The analysis was only performed within the involuntary group because there were not enough trials in which voluntary participants failed to recall an event ( $<9$ trials for each participant). Furthermore, only eight participants from the involuntary condition could be included in the analysis to ensure high statistical power; the remaining six had fewer than nine not recalled trials. The threshold for these initial analyses was lowered to $p<.05$ to reflect the small group size and the post hoc nature of the analysis. All activity is reported at a cluster size of 5 . The results from this analysis are shown in Table 3. 


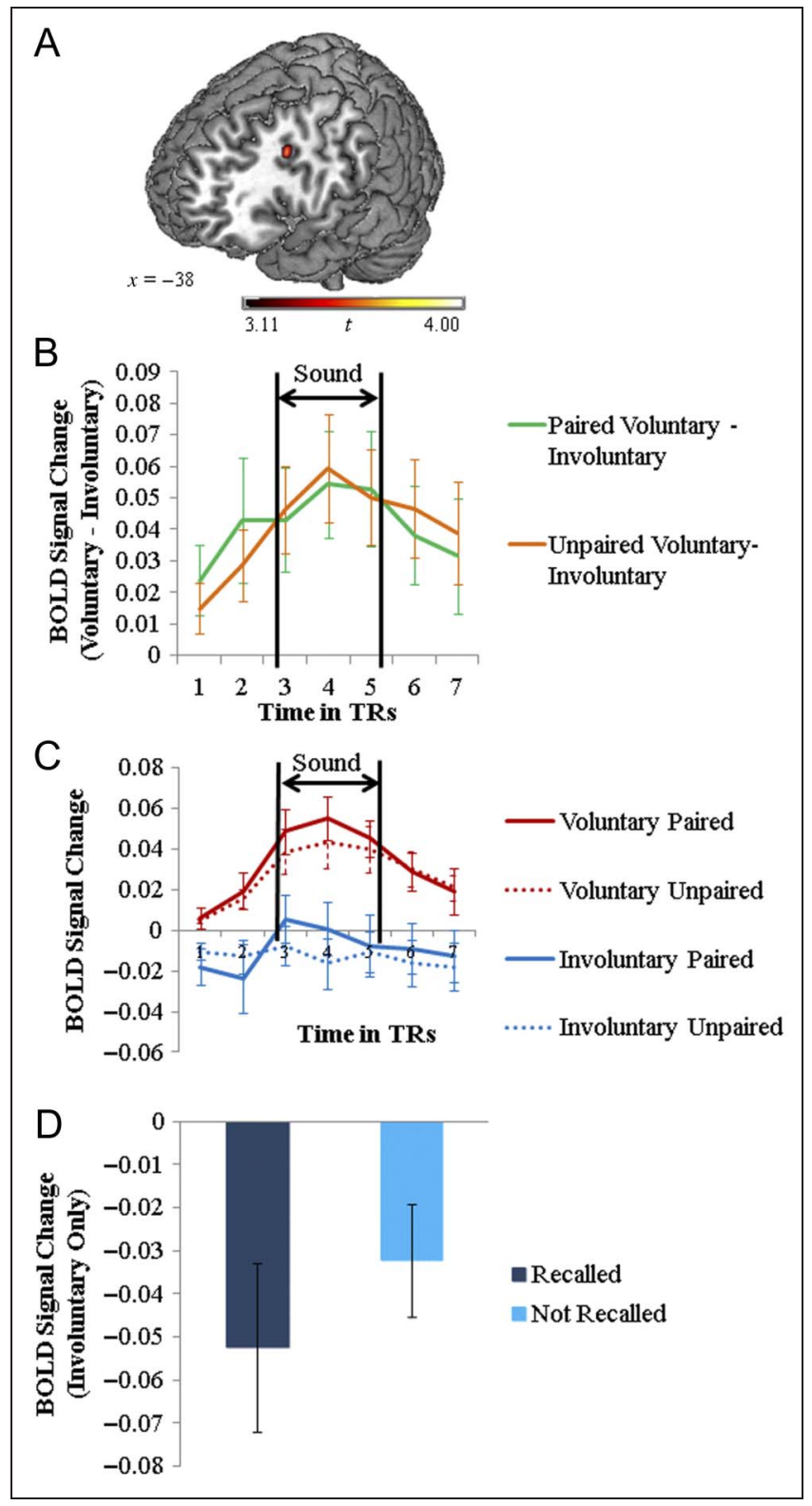

Figure 3. Effects of group on voluntary or involuntary memory recall. (A) Clusters showing differences between the voluntary and involuntary groups. The region shown is the left DLPFC. Brain images display significance at $p<.001,10$ voxels. (B) Graphs plot the estimated FIR response difference between voluntary and involuntary groups for the paired and the unpaired sounds within the DLPFC. The activity in the area between the two vertical lines indicates activity correlating with the peak of activity expected for the sound assuming a normal HDR. There is a significant difference between group (voluntary and involuntary groups) in this region but not condition (paired and unpaired sounds) (C) A graph plots the estimated FIR response for paired and unpaired sounds in the voluntary and the involuntary groups. (D) Activity within the DLPFC for the involuntary group for recalled trials compared with not recalled trials. There is no difference in activity within this region. This post hoc analysis was conducted at $p<.05$.

When we conducted an analysis using the clusters of activity from the Paired Status $\times$ Time interaction as a mask, we found right posterior cingulate, left parahippocampal gyrus, right precuneus, and a left lateralized cluster in superior parietal cortex, extending to inferior parietal cortex, to be active for recalled $>$ not recalled trials (Table 3, Figure 4). When we conducted an analysis using the frontal activity found in the main effect of Group analysis as a mask, we found no activity for recalled $>$ not recalled within the mask (Table 3; Figure 3D), supporting the earlier claim that dorsalolateral $\mathrm{pFC}$ does not contribute to the nonstrategic retrieval of mnemonic details or the retrieval of mnemonic details outside of a state of strategic retrieval. To further probe this result, we raised the threshold to $p<.01$ while retaining our initial cluster size of 10 . The results from this analysis with a raised threshold are reported in Table 4 . First, we looked within a mask comprising all frontal regions in the PickAtlas toolbox (www.fmri.wfubmc.edu/ download.htm) to examine whether there was activity anywhere in the frontal lobes. None was present. We then looked elsewhere in the brain and found activity overlapping with two of the four regions that are relevant to successful voluntary and involuntary retrieval, specifically visual regions, and posterior midline regions. These three regions are represented in bold in Table 4. In addition to these regions, we found activity in the cuneus and the parahippocampal gyrus (Table 4). Although a sample with enough power to use a more stringent threshold would be needed to confirm these results, they suggest that regardless of the control condition, involuntary memories do not elicit activity in frontal regions and they do elicit activity in regions typically associated with successful voluntary memory retrieval.

\section{DISCUSSION}

Our study yielded two main findings. First, voluntary and involuntary memories share considerable overlap in a network of regions strongly associated with successful episodic retrieval, including MTL (hippocampus, parahippocampal gyrus), posterior midline (retrosplenial and posterior cingulate cortices), VPC (angular gyrus), and sensory reactivation regions (visual cortex). Second, in contrast, voluntary memory was uniquely associated with activity in the left DLPFC, a region generally associated with cognitive control, and more specifically often found to be associated with controlled episodic retrieval. These findings represent strong evidence that voluntary and involuntary memories share the same basic neural structures with the primary difference being the difference in DLPFC activity. These findings are discussed in greater detail below.

\section{Paired versus Unpaired Sounds: Retrieval Success Network}

Voluntary and involuntary memories have many similarities. They have a similar distribution of emotional valence and intensity, they follow the same forgetting curve, and they occur with similar frequencies to each other at each stage of the life span (Berntsen \& Rubin, 2013; Berntsen, 2009; 
Table 3. Maxima of Regions for Recalled and Not Recalled within Involuntary Paired Sounds, Masked as Noted $(p<.05$, Cluster Size $=5$ )

\begin{tabular}{|c|c|c|c|c|c|c|c|c|}
\hline Region & $B A$ & Lat & Voxel Count & $x$ & $y$ & $z$ & $t$ & $p$ (Uncorrected) \\
\hline \multicolumn{9}{|l|}{ Recalled $>$ Not Recalled } \\
\hline \multicolumn{9}{|c|}{ Masked with Paired Status $\times$ Time activity } \\
\hline Posterior cingulate & 30 & $\mathrm{R}$ & 100 & 8 & -53 & 11 & 2.92 & .002 \\
\hline Parahippocampal gyrus & 30 & $\mathrm{~L}$ & & -8 & -41 & 4 & 2.72 & .004 \\
\hline Precuneus & 31 & $\mathrm{R}$ & & 11 & -60 & 23 & 2.04 & .022 \\
\hline Superior parietal lobule & 7 & $\mathrm{~L}$ & 12 & -26 & -68 & 46 & 2.42 & .009 \\
\hline \multicolumn{9}{|l|}{ Recalled $>$ Not Recalled } \\
\hline \multicolumn{9}{|c|}{ Masked with left frontal cortex main effect of group } \\
\hline No significant clusters & & & & & & & & \\
\hline
\end{tabular}

Rasmussen \& Berntsen, 2009; Rubin \& Berntsen, 2009; Berntsen \& Hall, 2004). Because of these behavioral findings, we predicted that there would also be overlapping regions of activity for voluntary and involuntary memories in regions typically associated with successful recall in voluntary memory studies.

Consistent with this first prediction, we found that both voluntary and involuntary groups showed paired-unpaired differences in regions strongly associated with successful episodic retrieval, including MTL (hippocampus, parahippocampal cortex), posterior midline (retrosplenial and posterior cingulate cortices), ventral parietal (angular gyrus), and sensory reactivation (visual cortex) regions (see Figure 2). These regions are commonly found in fMRI studies of recognition memory that compare activity for hits versus correct rejections or misses (for meta-analyses, see Kim, 2013; Spaniol et al., 2009).

Activations in the hippocampus and parahippocampal cortex have been linked to successful retrieval of contextual information or recollection, rather than to retrieval in the absence of contextual recollection or familiarity (for a review, see Diana, Yonelinas, \& Ranganath, 2007). Retrosplenial and posterior cingulate regions also tend to show greater activity for recollection than familiarity, consistent with their strong anatomical connections to the MTL (Daselaar, Fleck, \& Cabeza, 2006; Yonelinas, Otten, Shaw, \& Rugg, 2005). The current results are important because they indicate that the recruitment of these recollectionrelated MTL and posterior midline regions does not require the conscious intention to retrieve past events

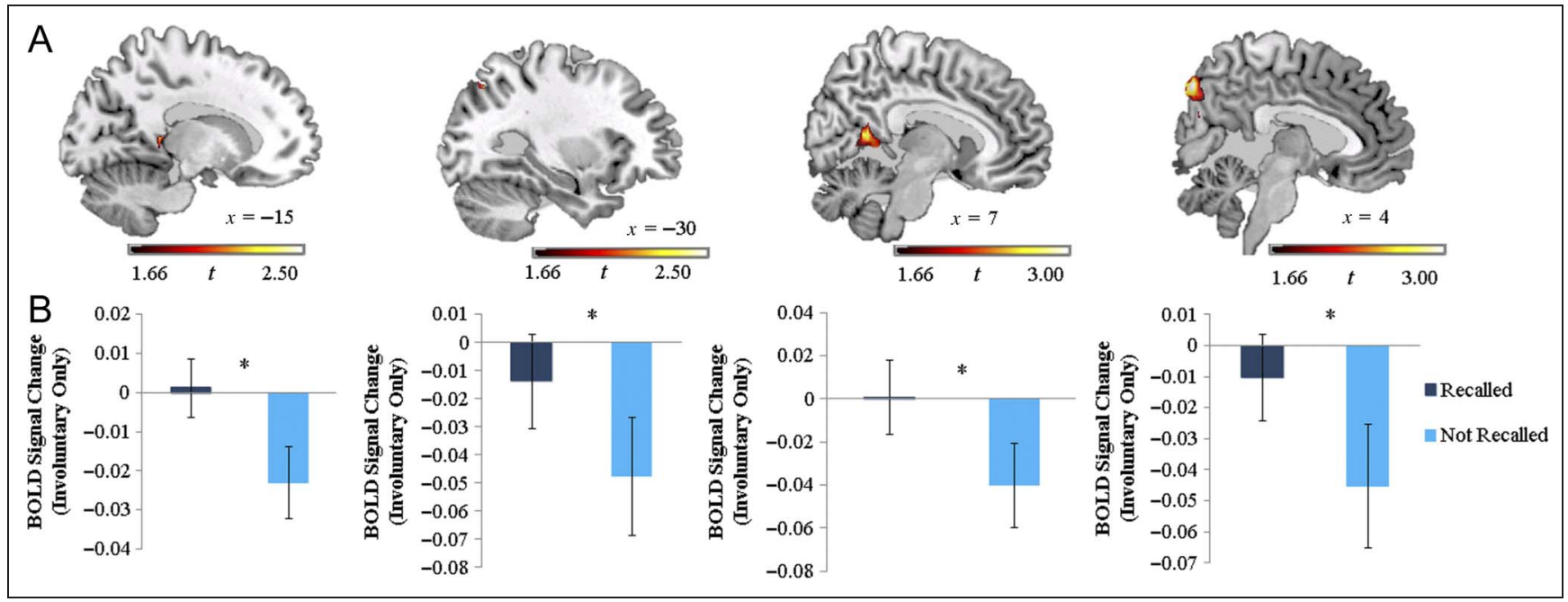

Figure 4. Regions showing activity within the involuntary group only for trials in which participants recalled a picture compared with trials in which participants did not recall a picture for paired sounds only. This post hoc analysis revealed a difference between these conditions at $p<.05$. (A) All regions overlap with regions in corresponding columns found in the Condition $\times$ Time interaction displayed in Figure 2 . From left to right, the regions are MTL, inferior parietal cortex, posterior midline regions, and visual regions. (B) Graphs showing the difference between recalled and not recalled trials. In all cases, there is greater activity in the recalled condition than the not recalled condition. 
but also occur when retrieval is involuntary and spontaneous. The finding that the hippocampus had similar activity for voluntary and involuntary memories fits very well with the hypothesis that the hippocampus is a module that automatically retrieves information in response to retrieval cues (Moscovitch, 1992, 1995).

The current finding that involuntary memory activates the MTL and posterior midline regions as much as voluntary memory is consistent with the results by Kompus et al. (2011) and Hall et al. (2008). As noted before, however, these previous studies used within-subject designs where the same participants performed both involuntary and voluntary memory tasks; hence, they could not control for the use of voluntary retrieval strategies during involuntary memory tasks. In the current study however, involuntary memory participants never performed an intentional retrieval task until the postscan questionnaire. Thus, our study provides the first clear evidence that the recruitment of the MTL and posterior midline regions is independent of the intention to retrieve episodic memory.

The VPC, particularly the angular gyrus, is another region that consistently shows greater activity for recollection than familiarity across fMRI studies (Ciaramelli et al., 2008; Vilberg \& Rugg, 2008). According to an episodic buffer hypothesis (Vilberg \& Rugg, 2008), this region mediates the maintenance of multimodal information within work- ing memory, where it is held available to access given the appropriate retrieval cue. Conversely, according to the attention to memory (AtoM) model (Cabeza et al., 2008), activity in VPC is driven by bottom-up attention processes, which are captured by recovered memories. Given that the voluntary retrieval of multimodal information and the maintenance of this information were requirements in the voluntary but not in the involuntary memory condition, the episodic buffer hypothesis would predict greater ventral parietal activity for voluntary memories, which were explicitly maintained, than involuntary memories, which were not (Vilberg \& Rugg, 2012). Thus, participants in the involuntary condition may have held such information in the episodic buffer, but such maintenance processes were not necessary in these individuals. In contrast, the AtoM model predicts similar ventral parietal activity for voluntary and involuntary memory because both capture attention bottom-up. Thus, although we cannot discount the possibility that participants in the involuntary condition engaged in covert elaborative voluntary retrieval, the current finding seems to be more consistent with the AtoM model than the episodic buffer hypothesis.

Finally, the finding of greater visual cortex activity for paired than unpaired sounds fits with previous fMRI evidence that sensory regions activated during encoding are reactivated during voluntary and involuntary retrieval (for a review, see Danker \& Anderson, 2010). In the cur-

Table 4. Maxima of Regions for Recalled and Not Recalled within Involuntary Paired Sounds, Unmasked $(p<.01$, Cluster Size $=10$ )

\begin{tabular}{|c|c|c|c|c|c|c|c|c|}
\hline Region & $B A$ & Lat & Voxel Count & $x$ & $y$ & $z$ & $t$ & $p$ (Uncorrected) \\
\hline \multicolumn{9}{|l|}{ Recalled $>$ Not Recalled } \\
\hline Precuneus & 7 & $\mathrm{R}$ & 25 & 4 & -79 & 46 & 3.30 & .001 \\
\hline Cuneus & 19 & $\mathrm{R}$ & 99 & 11 & -98 & 23 & 2.99 & .002 \\
\hline Precuneus & 31 & $\mathbf{L}$ & & -8 & -71 & 15 & 2.94 & .002 \\
\hline Cuneus & 17 & $\mathrm{~L}$ & & -8 & -83 & 11 & 2.91 & .002 \\
\hline Cuneus & 18 & $\mathrm{R}$ & & 15 & -83 & 19 & 2.91 & .002 \\
\hline Middle occipital Gyrus & 18 & $\mathbf{R}$ & & 11 & -98 & 11 & 2.86 & .003 \\
\hline Cuneus & 17 & $\mathrm{R}$ & & 8 & -94 & 4 & 2.81 & .003 \\
\hline Posterior cingulate & 30 & $\mathbf{R}$ & 15 & 8 & -53 & 11 & 2.92 & .002 \\
\hline Parahippocampal gyrus & 30 & $\mathrm{~L}$ & & -8 & -41 & 4 & 2.72 & .004 \\
\hline \multicolumn{9}{|l|}{ Not Recalled $>$ Recalled } \\
\hline Fusiform gyrus & 20 & $\mathrm{R}$ & 16 & 45 & -23 & -15 & 3.46 & .000 \\
\hline Anterior cingulate & 32 & $\mathrm{~L}$ & 20 & -4 & 26 & -8 & 3.37 & .001 \\
\hline Anterior cingulate & 32 & $\mathrm{R}$ & & 4 & 23 & -8 & 2.71 & .004 \\
\hline Anterior cingulate & 24 & $\mathrm{R}$ & & 4 & 30 & -4 & 2.51 & .007 \\
\hline
\end{tabular}

Bold regions indicate regions that overlap with regions found in the Paired Status $\times$ Time interaction.

$\mathrm{BA}=$ Brodmann's area Lat $=$ lateralization; $x y z=$ coordinates . 
rent study, participants encoded sound-picture pairs (paired sounds) or sound alone (unpaired sounds), and during retrieval, only sounds were presented. Thus, as in previous studies of visual reactivation (Wheeler et al., 2006; Wheeler \& Buckner, 2003, 2004; Vaidya, Zhao, Desmond, \& Gabrieli, 2002; Ishai, Ungerleider, \& Haxby, 2000; Wheeler, Petersen, \& Buckner, 2000; Kosslyn, Thompson, \& Alpert, 1997; Fletcher et al., 1995), our findings of activity in visual processing regions (e.g., superior occipital gyrus and precuneus) likely reflect the recovery of visual information. The new contribution of this study is that visual reactivation was similar for voluntary and involuntary memories, providing strong evidence that the reactivation can be bottom-up and does not require the intention to retrieve.

\section{Voluntary versus Involuntary Memory: Left DLPFC}

In addition to commonalities in retrieval success, there is also robust behavioral support for specific differences between voluntary and involuntary memories because of differences in retrieval (Berntsen, 2009). Consistent with our second prediction derived from these behavioral findings, we found that compared with involuntary memory, voluntary memory was associated with greater activity in lateral pFC. As illustrated by Figure 3, left DLPFC showed greater sustained activity in the voluntary than involuntary memory group for paired and unpaired sounds, suggesting a general state of strategic retrieval. The frequent association of this region with verbal working memory tasks and contextual reinstatement suggests that, in our task, the requirement for voluntary participants to maintain task goals and plan the associative retrieval put more demands on this region.

In fMRI studies of episodic retrieval, left DLPFC activity tends to increase as a function of the demands placed on controlled retrieval processes (Mitchell \& Johnson, 2009). The lateral pFC is also implicated in controlled and strategic cognitive processing (Koechlin, Ody, \& Kouneiher, 2003; Miller, 2000). Dobbins and collaborators (2002, 2003) found that left DLPFC (middle frontal gyrus) shows greater activity for source than item memory tasks and that this activity was similar for correct and incorrect source memory trials. According to these authors, this pattern suggests that left DLPFC mediates the attempt to retrieve episodic memory details (recollective attempt) rather than a process conditional on successful retrieval. This account fits well with the current finding that left DLPFC showed greater activity for voluntary than involuntary memory but did not differ between the condition in which retrieval success was extremely high (paired sounds: $94.50 \%$ picture recall) and the condition in which it was extremely low (unpaired sounds: $3.83 \%$ picture recall). The fact that this region was partly active during intertrial intervals (see activation time course in Figure 3) further suggests that this region may mediate not only trial-specific retrieval attempt processes but also more sustained strategic retrieval (Dobbins \& Han, 2006).

\section{Conclusion}

To summarize, we found that both voluntary and involuntary memories engaged the retrieval success network of MTL, posterior midline, ventral parietal, and sensory reactivation regions, whereas only voluntary memories recruited $\mathrm{pFC}$. These findings clarify the functions of these regions and have implications for the understanding of the neural mechanisms of both involuntary and voluntary memory retrieval. They also have clinical implications because involuntary memories are a symptom in many clinical disorders, including bipolar disorder, depression, social phobia, and posttraumatic stress disorder. Future studies may wish to further probe differences between voluntary and involuntary memories in people with clinical disorders as well as in populations with limited pFC resources, including children and older adults.

\section{Acknowledgments}

The authors thank the Danish National Research Foundation (DNRF93) and National Institute of Mental Health (grant R01 MH066079) for funding. We also thank Banafsheh Sharif-Askary and Sander Daselaar for their help.

Reprint requests should be sent to David C. Rubin, Department of Psychology and Neuroscience, Duke University Box 90086, Durham, NC 27708, or via e-mail: david.rubin@duke.edu.

\section{REFERENCES}

American Psychiatric Association. (2013). Diagnostic and statistical manual of mental disorders (5th ed.). Washington, DC: American Psychiatric Association.

Berntsen, D. (1996). Involuntary autobiographical memories. Applied Cognitive Psychology, 10, 435-454.

Berntsen, D. (2009). Involuntary autobiographical memories: An introduction to the unbidden past. New York: Cambridge University Press.

Berntsen, D. (2010). The unbidden past: Involuntary autobiographical memories as a basic mode of remembering. Current Directions in Psychological Science, 19, 138-142.

Berntsen, D., \& Hall, N. M. (2004). The episodic nature of involuntary autobiographical memories. Memory $\&$ Cognition, 32, 789-803.

Berntsen, D., \& Rubin, D. C. (2013). Involuntary memories and dissociative amnesia: Assessing key assumptions in PTSD research. Clinical Psychological Science, 2, 174-186.

Berntsen, D., Staugaard, S. R., \& Sørensen, L. M. (2013). Why am I remembering this now? Predicting the occurrence of involuntary (spontaneous) episodic memories. Journal of Experimental Psychology: General, 142, 426-444.

Buckner, R. L., \& Wheeler, M. E. (2001). The cognitive neuroscience of remembering. Nature Reviews Neuroscience, 2, 624-634.

Cabeza, R., Ciaramelli, E., Olson, I. R., \& Moscovitch, M. (2008). The parietal cortex and episodic memory: An attentional account. Nature Reviews Neuroscience, 9, 613-625. 
Cabeza, R., Dolcos, F., Graham, R., \& Nyberg, L. (2002). Similarities and differences in the neural correlates of episodic memory retrieval and working memory. Neuroimage, 16, 317-330.

Ciaramelli, E., Grady, C. L., \& Moscovitch, M. (2008). Top-down and bottom-up attention to memory: A hypothesis (AtoM) on the role of posterior parietal cortex in memory retrieval. Neuropsychologia, 46, 1828-1851.

Corbetta, M., Kincade, J. M., Ollinger, J. M., McAvoy, M. P., \& Shulman, G. L. (2000). Voluntary orienting is dissociated from target detection in human posterior parietal cortex. Nature Neuroscience, 3, 292-297.

Courtney, S. M., Ungerleider, L. G., \& Keil, K. (1997). Transient and sustained activity in a distributed neural system for human working memory. Nature, 386, 608-611.

Danker, J. F., \& Anderson, J. R. (2010). The ghosts of brain states past: Remembering reactivates the brain regions engaged during encoding. Psychological Bulletin, 136, 87-102.

Daselaar, S. M., Fleck, M. S., \& Cabeza, R. (2006). Triple dissociation in the medial temporal lobes: Recollection, familiarity, and novelty. Journal of Neurophysiology, 96, $1902-1911$.

D’Esposito, M., Postle, B. R., \& Rypma, B. (2000). Prefrontal cortical contributions to working memory: Evidence from event-related fMRI studies. Experimental Brain Research, 133, 3-11.

Diana, R. A., Yonelinas, A. P., \& Ranganath, C. (2007). Imaging recollection and familiarity in the medial temporal lobe: A three-component model. Trends in Cognitive Science, 11, 379-386.

Dobbins, I. G. (2001). The systematic discrepancy between $A^{\prime}$ for overall recognition and remembering: A dual-process account. Psychonomic Bulletin E Review, 8, 587-599.

Dobbins, I. G., Foley, H., Schacter, D. L., \& Wagner, A. D. (2002). Executive control during episodic retrieval: Multiple prefrontal processes subserve source memory. Neuron, 35, 989-996.

Dobbins, I. G., \& Han, S. (2006). Cue- versus probe-dependent prefrontal cortex activity during contextual remembering. Journal of Cognitive Neuroscience, 18, 1439-1452.

Dobbins, I. G., Rice, H. J., Wagner, A. D., \& Schacter, D. L. (2003). Memory orientation and success: Separable neurocognitive components underlying episodic recognition. Neuropsychologia, 41, 318-333.

Dobbins, I. G., \& Wagner, A. D. (2005). Domain-general and domain-sensitive prefrontal mechanisms for recollecting events and detecting novelty. Cerebral Cortex, 15, 1768-1778.

Donald, M. (1991). Origins of the modern mind: Three stages in the evolution of culture and cognition. Cambridge, MA: Harvard University Press.

Ebbinghaus, H. (1885). Über das Gedächtnis. Untersuchungen zur experimentellen Psychologie. Leipzig: Duncker \& Humblot.

Fleck, M. S., Daselaar, S. M., Dobbins, I. G., \& Cabeza, R. (2006). Role of prefrontal and anterior cingulate regions in decision-making processes shared by memory and nonmemory tasks. Cerebral Cortex, 16, 1623-1630.

Fletcher, P. C., Frith, C. D., Baker, S. C., Shallice, T., Frackowiak, R. S. J., \& Dolan, R. J. (1995). The mind's eye-Precuneus activation in memory-related imagery. Neuroimage, 2, 195-200

Habib, R., \& Nyberg, L. (2008). Neural correlates of availability and accessibility in memory. Cerebral Cortex, 18, 1720-1726.

Hall, N. M., Gjedde, A., \& Kupers, R. (2008). Neural mechanisms of voluntary and involuntary recall: A PET study. Behavioural Brain Research, 186, 261-272.
Hintzman, D. L. (2011). Research strategy in the study of memory: Fads, fallacies, and the search for the "coordinates of truth." Perspectives on Psychological Science, 6, 253-271.

Holmes, E. A., \& Bourne, C. (2008). Inducing and modulating intrusive emotional memories: A review of the trauma film paradigm. ACTA Psychologica, 127, 553-566.

Holmes, E. A., \& Mathews, A. (2010). Mental imagery in emotion and emotional disorders. Clinical Psychology Review, 30, 349-362.

Horowitz, M. J. (1986). Stress-response syndromes: A review of posttraumatic and adjustment disorders. Hospital $\varepsilon$ Community Psychiatry, 37, 241-249.

Ishai, A., Ungerleider, L. G., \& Haxby, J. V. (2000). Distributed neural systems for the generation of visual images. Neuron, 28, 979-990.

Kim, H. (2013). Differential neural activity in the recognition of old versus new events: An activation likelihood estimation meta-analysis. Human Brain Mapping, 34, 814-836.

Koechlin, E., Ody, C., \& Kouneiher, F. (2003). The architecture of cognitive control in the human prefrontal cortex. Science, 302, 1181-1185.

Kompus, K., Eichele, T., Hugdahl, K., \& Nyberg, L. (2011). Multimodal imaging of incidental retrieval: The low route to memory. Journal of Cognitive Neuroscience, 23, 947-960.

Kosslyn, S. M., Thompson, W. L., \& Alpert, N. M. (1997). Neural systems shared by visual imagery and visual perception: A positron emission tomography study. Neuroimage, 6, 320-334.

Lang, P. J., Bradley, M. M., \& Cuthbert, B. N. (2005). International Affective Picture System (IAPS): Affective ratings of pictures and instruction manual. Emotion. Gainesville, FL: NIMH, Center for the Study of Emotion and Attention.

Martin-Ordas, G., Berntsen, D., \& Call, J. (2013). Memory for distant past events in chimpanzees and orangutans Current Biology, 23, 1-4.

Meltzer, J. A., \& Constable, R. T. (2005). Activation of human hippocampal formation reflects success in both encoding and cued recall of paired associates. Neuroimage, 24, 384-397.

Miller, E. K. (2000). The prefrontal cortex and cognitive control. Nature Reviews Neuroscience, 1, 59-66.

Miller, G. A. (1962). Psychology, the science of mental life (1st ed.). New York: Harper \& Row.

Mitchell, K. J., \& Johnson, M. K. (2009). Source monitoring 15 years later: What have we learned from fMRI about the neural mechanisms of source memory? Psychological Bulletin, 135, 638-677.

Moscovitch, M. (1992). Memory and working-with-memory: A component process model based on modules and central systems. Journal of Cognitive Neuroscience, 4, $257-267$

Moscovitch, M. (1995). Recovered consciousness: A hypothesis concerning modularity and episodic memory. Journal of Clinical and Experimental Neuropsychology, 17, 276-290

Neisser, U. (1967). Cognitive psychology. East Norwalk, CT: Appleton-Century-Crofts.

O'Connor, A. R., Han, S., \& Dobbins, I. G. (2010). The inferior parietal lobule and recognition memory: Expectancy violation or successful retrieval? The Journal of Neuroscience, 30, 2924-2934

Preuschhof, C., Heekeren, H. R., Taskin, B., Schubert, T., \& Villringer, A. (2006). Neural correlates of vibrotactile working memory in the human brain. The Journal of Neuroscience, 26, 13231-13239. 
Rasmussen, A. S., \& Berntsen, D. (2009). Emotional valence and the functions of autobiographical memories: Positive and negative memories serve different functions. Memory E Cognition, 37, 477-492.

Rasmussen, A. S., \& Berntsen, D. (2011). The unpredictable past: Spontaneous autobiographical memories outnumber autobiographical memories retrieved strategically.

Consciousness \& Cognition, 20, 1842-1846.

Rubin, D. C., \& Berntsen, D. (2009). The frequency of voluntary and involuntary autobiographical memories across the life span. Memory \& Cognition, 37, 679-688.

Schank, R. C. (1999). Dynamic memory revisited (2nd ed.). New York: Cambridge University Press.

Spaniol, J., Davidson, P. S., Kim, A. S., Han, H., Moscovitch, M., \& Grady, C. L. (2009). Event-related fMRI studies of episodic encoding and retrieval: Meta-analyses using activation likelihood estimation. Neuropsychologia, 47, 1765-1779.

Steel, C., Fowler, D., \& Holmes, E. A. (2005). Trauma-related intrusions and psychosis: An information processing account. Behavioural and Cognitive Psychotherapy, 33, 139-152.

Thompson-Schill, S. L., D’Esposito, M. D., Aguirre, G. K., \& Farah, M. J. (1997). Rose of left inferior prefrontal cortex in retrieval of semantic knowledge: A reevaluation. Proceedings of the National Academy of Sciences, 94, 14792-14797.

Vaidya, C. J., Zhao, M., Desmond, J. E., \& Gabrieli, J. D. E. (2002). Evidence for cortical specificity in episodic memory: Memory-induced re-activation of picture processing areas. Neuropsychologia, 40, 2136-2143.
Vilberg, K. L., \& Rugg, M. D. (2008). Memory retrieval and the parietal cortex: A review of evidence from a dual-process perspective. Neuropsychologia, 46, 1787-1799.

Vilberg, K. L., \& Rugg, M. D. (2012). The neural correlates of recollection: Transient versus sustained fMRI effects. The Journal of Neuroscience, 32, 15679-15687.

Wagner, A. D., Shannon, B. J., Kahn, I., \& Buckner, R. L. (2005). Parietal lobe contributions to episodic memory retrieval. Trends in Cognitive Sciences, 9, 445-453.

Wheeler, M. E., \& Buckner, R. L. (2003). Functional dissociations among components of remembering: Control, perceived oldness, and content. The Journal of Neuroscience, 23, 3869-3880.

Wheeler, M. E., \& Buckner, R. L. (2004). Functional-anatomic correlates of remembering and knowing. Neuroimage, 21, 1337-1349.

Wheeler, M. E., Petersen, S. E., \& Buckner, R. L. (2000). Memory's echo: Vivid remembering reactivates sensory-specific cortex. Proceedings of the National Academy of Sciences, 97, 11125-11129.

Wheeler, M. E., Shulman, G. L., Buckner, R. L., Miezin, F. M., Velanova, K., \& Petersen, S. E. (2006). Evidence for separate perceptual reactivation and search processes during remembering. Cerebral Cortex, 6, 949-959.

Williams, A. D., \& Moulds, M. L. (2010). The impact of ruminative processing on the experience of self-referent intrusive memories in dysphoria. Behavior Therapy, 41, 38-45.

Yonelinas, A. P., Otten, L. J., Shaw, K. N., \& Rugg, M. D. (2005). Separating the brain regions involved in recollection and familiarity in recognition memory. The Journal of Neuroscience, 25, 3002-3008. 